Khaja M, et al. Ann Case Report 6: 719.

www.doi.org/10.29011/2574-7754.100719

www.gavinpublishers.com

Case Series

GAVIN PUBLISHERS

\title{
Thoracentesis: Common Bedside Procedure
}

\section{Misbahuddin Khaja ${ }^{1^{*}}$, Alla Yugay ${ }^{2}$, Shoaib Ashraf ${ }^{3}$}

${ }^{1}$ Attending Physician, Division of Pulmonary and Critical Care Medicine, Bronx Care Health System, Affiliated with Icahn School of Medicine at Mount Sinai 1650 Grand Concourse, Bronx, NY 10457, USA

${ }^{2}$ Second Year Pulmonary Fellow, Division of Pulmonary and Critical Care Medicine, Bronx Care Health System, Affiliated with Icahn School of Medicine at Mount Sinai 1650 Grand Concourse Bronx, NY 10457, USA

${ }^{3}$ Third Year Medical Resident, Department of Medicine, Bronx Care Health System, Affiliated with Icahn School of Medicine at Mount Sinai 1650 Grand Concourse Bronx, NY 10457, USA

*Corresponding author: Misbahuddin Khaja, Attending Physician, Division of Pulmonary and Critical Care Medicine, Bronx Care Health System, Affiliated with Icahn School of Medicine at Mount Sinai 1650 Grand Concourse, Bronx, NY 10457, USA.

Citation: Khaja M, Yugay A, Ashraf S (2021) Central Line: Common Bedside Procedure. Ann Case Report 6: 719. DOI: 10.29011/2574-7754.100719

Received Date: 20 December 2021; Accepted Date: 27 December 2021; Published Date: 30 December 2021

\begin{abstract}
Thoracentesis is a bedside procedure that is done under ultrasound guidance to remove fluid from pleural space. It is performed for both diagnostics and therapeutics. Pleural fluid analysis can differentiate between transudate and exudative effusion. There are no absolute contraindications, but few relative contraindications are coagulopathy and insertion site infection. After the informed consent position, the patient leaning forward and prepare the insertion site with chlorhexidine. A local anesthetic should be administered, a needle should be inserted above the rib edge, and fluid is sent for analysis. The use of ultrasound-guided thoracentesis lowers the risk of complications and localizes the puncture site and pleural fluid characteristics. Although it is considered a low-risk procedure, complications of thoracentesis, including pneumothorax, bleeding, and reexpansion pulmonary edema, can lead to increased morbidity and mortality. Thoracentesis is a procedural skill that can be performed by emergency, pulmonary critical care, and hospitalist physician.
\end{abstract}

\section{Introduction}

Thoracentesis is a minimally invasive bedside procedure performed in outpatient and inpatient settings in which we remove the excess fluid from the pleural space. It is performed for both diagnostics and therapeutic reasons in the appropriate clinical settings. The procedure usually takes fifteen to twenty minutes under imaging guidance to be visualized and rapidly sample the pleural fluid content [1]. A wide range of co-morbidities can cause pleural effusions, i.e., heart failure, pneumonia, cancer, or fluid may leak from the peritoneal cavity causing the pleural effusion [2]. Clinicians suspect pleural effusion during a physical examination can be diagnosed with chest X-ray, bedside chest ultrasound, or computed tomography (CT) scan of the chest. This topic will review the indications, contraindications, procedures of thoracentesis, and its complications.

\section{Indication}

Thoracentesis is generally not required in patients with exceedingly small pleural effusion, or etiology is obvious, know diagnosis with no long-term implication like viral pleurisy, but most new effusions require thoracentesis. Therefore, it is indicated for both diagnostic and therapeutic clinical management of pleural effusion $[2,3]$.

- To diagnose tuberculosis, Para pneumonic effusion.

- Large volume therapeutic thoracentesis for symptomatic relief.

- Initial diagnoses of malignant pleural effusion.

- $\quad$ Trapped Lung. 
- Recurrent malignant pleural effusion after pleurodesis as palliative thoracentesis.

- Hemothorax, Pneumothorax or Chylothorax.

Typically, 20-50 cc of pleural fluid is removed for laboratory studies in diagnostic thoracentesis, but one and a half liters of fluid can be removed in therapeutic thoracentesis for symptomatic relief and cytology sampling.

\section{Contraindication}

Thoracentesis is a minimally invasive procedure without any absolute contraindications but few relative contraindications worth mentioning $[4,5]$ :

- Coagulopathy, INR more than two.

- Thrombocytopenia, i.e., less than $50,000 / \mathrm{mm}^{3}$.

- Serum creatinine $>6 \mathrm{mg} / \mathrm{dl}$.

- Insufficient pleural fluid $<1 \mathrm{~cm}$ adjacent to the parietal pleura.

- Bullous lung disease, severely decreased lung function, Single-lung patient.

- Infection at insertion site including cellulitis or Herpes zoster over the entry site.

\section{Procedure}

Thoracentesis is a minimal invasive bedside procedure that takes about fifteen to twenty minutes under imaging guidance. Bedside ultrasound is the most common imaging modality used for the procedure. Following are the steps for the thoracentesis procedure $[2,6,7]$.

- Informed consent from patient/family after explaining the procedure.

- Position the patient, sitting, arms resting on the table, leaning forward.

- Auscultate and percuss the chest wall to determine the height of the effusion, followed by an ultrasound to confirm the location.

- Ultrasound is used to locate the site for needle insertion. It improves not only the safety of the procedure but also accuracy. If the patient moves, then the site has to be located and marked again with help of an ultrasound.

- Prepare the confirmed site and sterilize with chlorohexidine and drape the patient.

- Local anesthetic ( $1 \%$ lidocaine) should be administered.

- The examiner determines the best angle for needle insertion that will access the fluid and avoid adjacent organs. The transducer angle defines the angle at which the needle/ syringe assembly will be held at the time of actual needle advancement. Slide the needle over the superior aspect of the rib while intermittently aspirating and injecting perpendicular to the pleural.

- A syringe attached to a small gauge needle 20- to 22-gauge and loaded with 1 or 2 percent lidocaine. It is inserted through the skin and toward the upper border of the rib along the trajectory defined by the ultrasonography examination.

- Once the rib is reached, anesthesia should be administered to the needle, cephalad over the rib, through the parietal pleura, and into the pleural space. Return of pleural fluid indicates that the needle has entered the pleural space confirms the distance from the skin to the pleural fluid collection.

- Once the selected insertion site is anesthetized, the operator makes a cut in through the epidermis using the scalpel blade. The cut is made with vertical movement of the scalpel blade using a stabbing action. The cut is through the full thickness of the epidermis.

- Generation over-the-needle catheters have a three-way stopcock permanently affixed to the catheter. The stopcock is pre-orientated in a position that is closed to the patient; with this type of system, when the needle is removed from the stopcock, there is no risk of pneumothorax from entrainment of air into the pleural space during inspiration. Older design catheter over needle devices required attachment of the stopcock to the catheter following insertion of the catheter into the pleural space; this type of system could result in the entrainment of air. We recommend using only the former for improved safety of the procedure.

- With the stopcock attached, the over-the-needle catheter is inserted through the skin while continuous negative pressure is applied to the syringe. When fluid is aspirated, the needle is inserted another $5 \mathrm{~mm}$ to ensure that the needle and the catheter are positioned within the pleural fluid collection. The needle introducer is held stationary while the catheter is pushed forward over the needle to the desired depth or until the catheter hub is against the skin (whichever comes first). The needle is then removed (through the stopcock), and the catheter and stopcock remain in place.

- If pleural pressure is to be measured to evaluate possible trapped or entrapped lung, then the manometer can be attached to the side port of the stopcock.

- Generation over-the-needle catheter device kits include a drainage tubing set that simplifies therapeutic thoracentesis. One-way valves are positioned in the tubing set such that the operator may draw fluid into the $50 \mathrm{~mL}$ syringe and then 
push it out into the collection bag. When properly attached, the tubing set is a closed system. It offers the advantage of a convenient means of fluid drainage with no risk of inadvertent air entrainment into the pleural space during fluid removal.

- More fluid may be removed if required for additional tests.

- Standard tests on pleural fluid samples include $\mathrm{pH}$, cell count, glucose, protein, culture, lactate dehydrogenase, amylase, gram stain, and cytology.

- For pH measurement, a fresh $3 \mathrm{~mL}$ syringe pre-coated with heparin $(<0.4 \mathrm{~mL}$ or approximately eight units) or a $3 \mathrm{~mL}$ arterial blood gas syringe that comes pre-coated with heparin is attached to the thoracentesis catheter to draw pleural fluid directly from the patient. Any air bubbles in the syringe should be immediately expelled from the syringe and the syringe placed on ice. A blood gas analyzer should analyze fluid within one hour of collection.

- Once a sample of fluid is removed for analysis, there are three methods to remove additional fluid (if desired), Gravity Drainage, Syringe, Vacuum Bottle.

- Post-procedure, order chest $\mathrm{x}$-ray, monitor blood pressure and respiration status to ensure patients do not have complications.

- Post-procedure ultrasound is used to document lung sliding, if present, indicate the absence of pneumothorax.

\section{Complications}

Although generally considered a low-risk intervention, we do come across complications of thoracentesis $[8,9,10]$.

- Pneumothorax.

- Hematoma, hemothorax, or hemoperitoneum.

- Soft tissue infection.

- Empyema.

- Rare complications include spleen or liver puncture.

- Vasovagal events.

- Tumor metastasis via seeding the needle tract.

- Anesthetic adverse reactions.

- Shortness of breath, cough, and re-expansion pulmonary edema.

\section{Conclusion}

Thoracentesis is a minimally invasive bedside procedure performed in both the inpatient and outpatient settings. The most common indication is establishing the cause of pleural effusion when unexplained fluid accumulates in the pleural space. Although it is considered a low-risk procedure, complications occur after the procedure, including pneumothorax, chest wall hematoma, hemothorax, and re-expansion pulmonary edema, leading to increased morbidity and mortality. The use of bedside ultrasound minimizes complications and improves the safety the accuracy of the procedure.

\section{Acknowledgments}

No funding was provided for the production of this paper.

\section{Disclosures}

None of the authors has a financial relationship with a commercial entity that has an interest in the subject of the manuscript. No financial support was used for this case series.

\section{Authors Contributions}

M Khaja, A Alapati and S Ashraf searched the literature and wrote the manuscript. M Khaja conceived and edited the manuscript. All authors have made significant contributions to the manuscript and have reviewed it before submission. All authors have confirmed that the manuscript is not under consideration for review at any other Journal. All authors have read and approved the final manuscript.

\section{References}

1. Feller-Kopman, D (2006) Ultrasound-guided thoracentesis. Chest. 129: $1709-1714$

2. Roberts, J.R. and J.R. Hedges, Roberts and Hedges' (2013) Clinical procedures in emergency medicine E-book. 2013: Elsevier Health Sciences.

3. J W Sokolowski Jr, L W Burgher, F L Jones Jr, J R Patterson, P A Selecky (1989) Guidelines for thoracentesis and needle biopsy of the pleura. This position paper of the American Thoracic Society was adopted by the ATS Board of Directors, June 1988 Am Rev Respir Dis.140: 257-8.

4. McVay, P.A, P.T. Toy (1991) Lack of increased bleeding after paracentesis and thoracentesis in patients with mild coagulation abnormalities. Transfusion. 31: 164-71.

5. Rebecca M Hibbert, Thomas D Atwell, Alexander Lekah, Maitray D Patel, Rickey E Carter (2013) et al. Safety of ultrasound-guided thoracentesis in patients with abnormal preprocedural coagulation parameters. Chest. 144: 456-463.

6. Thomsen, T.W, J. DeLaPena, G.S. Setnik (2006) Videos in clinical medicine. Thoracentesis. The New England journal of medicine. 355: e16.

7. DeBiasi, E.M. and J. Puchalski (2016) Thoracentesis: state-of-theart in procedural safety, patient outcomes, and physiologic impact. Pleura. 3: 2373997516646554.

8. Mercaldi, C.J, S.F. Lanes (2013) Ultrasound guidance decreases complications and improves the cost of care among patients undergoing thoracentesis and paracentesis. Chest. 143: 532-538. 
Citation: Khaja M, Yugay A, Ashraf S (2021) Central Line: Common Bedside Procedure. Ann Case Report 6: 719. DOI: 10.29011/25747754.100719

9. Craig E Gordon, David Feller-Kopman, Ethan M Balk, Gerald W Smetana (2010) Pneumothorax following thoracentesis: a systematic review and meta-analysis. Arch Intern Med. 170: 332-9.
10. Eric P CanteY, James M Walter, Thomas Corbridge (2016) Jeffrey H BarsukComplications of thoracentesis: incidence, risk factors, and strategies for prevention. Current opinion in pulmonary medicine. 22: 378-385. 